\title{
Travailler sur l'islam dans la Bosnie en guerre.
}

\section{Partie 1}

\section{Xavier Bougarel}

\section{(2) OpenEdition}

1 Journals

\section{Édition électronique}

URL : http://journals.openedition.org/conflits/821

DOI : $10.4000 /$ conflits.821

ISSN : 1777-5345

Éditeur :

CCLS - Centre d'études sur les conflits lilberté et sécurité, L'Harmattan

\section{Édition imprimée}

Date de publication : 1 septembre 2002

ISBN : 2-7475-3432-4

ISSN : 1157-996X

\section{Référence électronique}

Xavier Bougarel, «Travailler sur l'islam dans la Bosnie en guerre. Partie 1 », Cultures \& Conflits [En ligne], 47 | automne 2002, mis en ligne le 29 avril 2003, consulté le 30 mars 2021. URL : http:// journals.openedition.org/conflits/821; DOI : https://doi.org/10.4000/conflits.821

Ce document a été généré automatiquement le 30 mars 2021.

Creative Commons License 


\title{
Travailler sur l'islam dans la Bosnie en guerre. Partie 1
}

\author{
Xavier Bougarel
}

J'ai écrit ce texte à la demande de Valérie Amiraux, à qui je dois de m'avoir poussé dans un avion Istanbul-Paris, un jour où je n'avais même plus la force de le faire. Ce texte est étrange, y compris à mes propres yeux. Il ne demandait sans doute qu'à être écrit depuis de longues années. Ce n'est pas un texte scientifique, même s'il traite de l'activité scientifique. Il se situe quelque part entre la remémoration onirique, la confession et la déclaration de guerre. Ce mélange des genres explique sans doute son aspect parfois confus ou ésotérique, certains dérapages de vocabulaire et certaines maladresses de style que je tiens à conserver, malgré les malentendus qu'ils peuvent susciter et les blessures qu'ils pourraient rouvrir.

1 Le siège de Sarajevo, le château de Prague et les journées de Barcelone

2 Quand sont évoquées les difficultés d'une recherche sur un pays en guerre, c'est d'abord la prise de risque, la confrontation à la menace des armes qui viennent à l'esprit. Pourtant, dans mon cas, la vraie difficulté n'est pas là. D'une part, j'ai très vite compris que je n'avais pas ce goût de l'adrénaline qui fait les bons reporters de guerre. Pendant les trois ans et demi qu'a duré le conflit bosniaque (avril 1992-décembre 1995), j'ai donc évité avec soin de me retrouver dans des situations périlleuses, et adapté en conséquence mes façons de faire. D'autre part, vu sa proximité géographique, sa forte médiatisation et, plus encore, ses liens indéniables mais complexes avec la référence morale fondatrice qu'est devenu l'Holocauste, la guerre en Bosnie-Herzégovine a avant tout constitué, pour celles et ceux qui s'y sont confrontés de l'extérieur, une épreuve morale ${ }^{1}$.

3 C'est surtout à cette confrontation morale, et donc abstraite avec la guerre que je souhaite m'intéresser ici. Pour ce faire, je tenterai d'abord d'expliquer comment les circonstances de mon travail sur la Bosnie en guerre, les aspects de cette réalité auxquels je me suis intéressé, ont suscité un certain nombre de chocs et de dilemmes moraux, de ruptures et d'impasses personnelles². Je tâcherai aussi de montrer en quoi cette expérience particulière de la guerre a influencé mes propres travaux, mes 
rapports avec d'autres acteurs présents sur le terrain et, finalement, déterminé certains choix.

Préalablement, et en espérant ne pas sombrer dans l'introspection, il me paraît utile de préciser un certain nombre de points. Ma confrontation à la guerre en BosnieHerzégovine a été précédée par un engagement militant relativement long et intensif, et marqué par une double source d'inspiration: le mouvement anarchiste et anarchosyndicaliste d'une part, la dissidence est-européenne d'autre part, les deux se rejoignant au travers de concepts tels que la « société contre l'Etat " (Pierre Clastres) et l'« anti-politique » (György Konrad), la « culture de soi-même » (Fernand Pelloutier) et la « vie dans la vérité » (Vaclav Havel). Il va sans dire que les tristes réalités de l'Europe post-communiste se chargèrent d'ébranler ce charmant édifice. Ainsi, quand les vertus libératrices que l'anarchisme attribue à la désintégration de l'Etat, au surgissement des milices et au fracas des armes se trouvèrent confrontées à l'éclatement sanglant de la Yougoslavie. Ainsi, également, quand Vaclav Havel, apôtre de la «force des faibles », se fit celui des missiles de l'OTAN, parachevant de la sorte la reconversion des anciens dissidents en politiciens raisonnables.

5 Par chance, l'hommage à la Catalogne de Georges Orwell, placé dans mes bagages lors de mon départ pour Belgrade en décembre 1990, m'avait in extremis préparé à la réalité sordide de la guerre ${ }^{3}$. Par chance aussi, bien des années plus tard, Tzvetan Todorov m'apprit, face à l'extrême, à redéployer mes inquiétudes dans des engagements discrets et personnels ${ }^{4}$. Il reste pourtant de cette période quelques questions sans réponses: comment un jeune étudiant anarchiste, à peine expulsé de son milieu politique pour des raisons ridicules, peut-il se retrouver consultant pour les ministères des Affaires étrangères et de la Défense? Comment se résoudre à la nécessité d'une intervention militaire en Yougoslavie, sans perdre de vue une raison essentielle et trop souvent oubliée de son éclatement sanglant, à savoir la militarisation de sa société et le surdimensionement de son appareil militaire? Enfin, comment formuler cette troublante et sans doute indépassable ambivalence du politique, qui veut que l'Etat contienne la violence, au double sens du verbe « contenir »?

6 Mais là n'est pas l'essentiel, pour ce qui nous intéresse ici. Ce qui explique mon expérience propre de la guerre en Bosnie-Herzégovine, et les positionnements intellectuels ou moraux qui en découlent, c'est sans doute moins mon bagage militant que les moments, les lieux, les angles d'attaque à partir desquels je l'ai appréhendée. Il s'en est peut-être fallu de peu, en effet, pour que je me retrouve aux côtés de ceux qui manifestaient pour la Bosnie, faisaient d'Alija Izetbegović l'emblème de la lutte antifasciste, partaient à Sarajevo apporter leur soutien et ressourcer leur foi. A ceux que j'avais parfois côtoyés dix ans auparavant dans le mouvement de soutien au syndicat Solidarnosc, j'expliquais notre désaccord par la formule suivante : « Vous êtes allés vers la guerre, la guerre est venue vers moi». Une chose en particulier m'a toujours mis mal à l'aise chez les militants revenant de Sarajevo: ils paraissaient chargés de sens, renforcés dans leur indignation et dans leurs certitudes. Quand, pour ma part, je suis rentré de Yougoslavie en juin 1992, je me suis aperçu que j'avais perdu là-bas toute capacité de révolte, et toute conviction un tant soit peu structurée. Pour diverses raisons - manque de courage, sentiment de culpabilité et autres -, je ne suis pas retourné à Sarajevo pendant la guerre. Mais les quelques incursions prudentes que j'ai faites dans la Bosnie en guerre se sont toujours soldées par un profond malaise, une 
irrépressible nausée, et un retour prématuré. Pour cette raison au moins, nous n'avions pas grand chose à nous dire.

7 C'est le hasard qui a placé la guerre sur ma route, et celle-ci m'est tombée dessus sans que j'y sois vraiment préparé. En 1990, devant effectuer mon service national dans le cadre de la coopération, j'ai finalement préféré partir à Belgrade plutôt qu'à Varsovie, car le poste qui m'y était proposé - lecteur de français à la Faculté de droit - me paraissait plus tranquille. Frais émoulu de Sciences Po, j'y avais découvert sans l'avoir choisi le monde étrange de l'islam. Sur les conseils de Rémy Leveau, un de mes professeurs, j'ai alors décidé d'utiliser mon séjour à Belgrade pour étudier les musulmans de Bosnie-Herzégovine. Au simple fait que la guerre soit venue vers moi, s'ajoutent donc d'autres circonstances qui ont façonné mon expérience propre des guerres yougoslaves.

Dans le Belgrade que j'ai découvert en décembre 1990, la guerre paraissait encore si irréelle, alors qu'elle était si proche. Je l'ai vue prendre forme et monter en puissance, tout en m'y habituant un peu plus chaque jour. Quand, de retour dans le Paris oisif de l'été 1992, je m'étonnais de m'indigner si peu des camps ou des viols que tous découvraient avec horreur, il me fallait remonter dans ma mémoire pour comprendre : ces camps, ces viols, je les avais acceptés depuis quand ? Le jour où, descendant du bus place de la Révolution, je ne m'étais pas arrêté pour savoir qui figurait sur cette photo entourée de noir, et devant laquelle les Belgradois déposaient des cierges, des oranges ou des paquets de cigarettes? Le jour où, lisant mon si cher quotidien « Borba $»^{5}$ au petit déjeuner, j'avais tourné avec ennui cette page qui annonçait une fois encore des morts, là-bas, en Krajina ou en Slavonie orientale ? Le jour où, écoutant le concierge de la Faculté de droit me raconter ses exploits militaires, j'avais mis la cendre de ma cigarette dans une douille d'obus marquée « Vukovar, octobre 1991 »?

Certes, j'ai fréquenté un temps les maigres rassemblements pacifistes de Belgrade, mais je me suis vite lassé de ces enfants de la bourgeoisie rouge qui rachetaient leur âme en invoquant l'Europe. Dans les années 1980, j'avais connu la clandestinité polonaise et les milieux pacifistes est-allemands, et je ne retrouvais pas dans la "dissidence" yougoslave le même souci moral, la même acceptation de certains sacrifices personnels. Militant d'extrême-gauche rôdé à l'action syndicale, je ne comprenais pas pourquoi, à de rares exceptions près, les pacifistes belgradois ignoraient superbement ces milliers de déserteurs qui quittaient le front en masse, et se rassemblaient devant leurs mairies de province en exigeant leur démobilisation ${ }^{6}$. A ma grande surprise, je me sentais finalement mieux avec ceux qui, feignant de croire que tout cela ne les concernait pas, se contentaient de préserver leur santé mentale, qui en cultivant sa passion pour la peinture ou l'archéologie, qui en partant randonner en montagne, qui en pique-niquant sur les bords du Danube, à deux pas de la base aérienne de Batajnica, avec sa ronde incessante de Migs et d'hélicoptères de combat ${ }^{7}$.

10 Car, bien sûr, si j'ai pu si facilement m'habituer à la guerre, c'est qu'elle ne s'est jamais emparée de ma vie quotidienne. Assis sur les bords du Danube, nous savions que les bombes portées par les Migs ne nous étaient pas destinées, et le simple fait de prendre l'air n'a jamais signifié à Belgrade risquer sa vie en s'exposant au tir des snipers. Pour m'en rendre compte, il m'a là encore fallu du temps ou, plus précisément, d'autres lieux, d'autres situations. Quand je suis retourné pour la première fois en Croatie, je n'ai pas saisi le sens de ces mots apparus sur l'écran de télévision: "Opća opasnost ", "Alerte générale », car j'ignorais ce qu'est un bombardement d'artillerie. Quand je suis 
arrivé pour la première fois à Tuzla, ville à travers laquelle j'avais choisi de découvrir la Bosnie en guerre, ma logeuse m'a gentiment attribué sa chambre la mieux orientée, celle par la fenêtre de laquelle aucun obus ne pouvait rentrer. Il m'a alors fallu un bon moment avant de me décider à ressortir dans la rue. Le fait de passer de l'autre côté du front m'a brutalement ouvert les yeux sur le fait que, en dépit de mes élucubrations savantes, mon expérience de la guerre était profondément, irrémédiablement serbe. Il m'a ensuite fallu beaucoup de temps pour l'admettre, et pour comprendre en quoi cette expérience représentait, elle aussi, une source valable d'inspiration et de réflexion.

11 De décembre 1990 à avril 1992, du reste, mes divers séjours à Sarajevo m'ont appris que les Sarajéviens et les Belgradois n'avaient pas, à l'époque, une perception radicalement différente de la guerre. Certes, le mouvement anti-guerre était beaucoup plus fort à Sarajevo qu'à Belgrade, en même temps que la peur d'être emporté par la tourmente ${ }^{8}$. Mais, comme les Belgradois, les Sarajéviens continuaient à vaquer à leurs occupations, regardaient les images de Vukovar et de Dubrovnik comme une sorte de spectacle étrange et lointain, espéraient secrètement que tout ceci n'était qu'un mauvais rêve. Les uns et les autres n'ont cessé de pouvoir se comprendre qu'au printemps 1992, quand les premières barricades sont apparues dans les faubourgs de Sarajevo, quand les kalachnikovs se sont mises à crépiter, la nuit, sur les collines, quand les premiers obus sont tombés sur la ville.

J'étais à Sarajevo en mars 1992, pour assister au référendum sur l'indépendance puis, de nouveau, quelques jours plus tard, pour participer au mois de ramadan. Parmi les souvenirs que j'ai de ces semaines fatidiques, certains me reviennent souvent à l'esprit.

$\mathrm{Au}$ lendemain du référendum sur l'indépendance, la ville se réveilla dans un étrange silence, que déchirait parfois la sirène d'un véhicule de police ou une rafale de kalachnikov. Les voitures et les tramways n'envahissaient plus le boulevard Maréchal Tito sur lequel donnaient nos fenêtres ${ }^{9}$, car les barricades érigées dans la nuit bloquaient toute circulation. Sortis en quête d'un hypothétique petit déjeuner, nous croisâmes sur la rue piétonne Vase Miškina, quasi-déserte, un homme qui avançait nu, les bras écartés et le regard perdu devant lui. Des policiers, kalachnikov sous le bras, le regardaient passer en riant. Revenus dans la famille où nous logions, la mère me demanda si je pensais qu'il y aurait la guerre, et s'il fallait partir. Je lui répondis que je ne savais pas.

De retour à Sarajevo quelques jours plus tard, je sympathisais avec certains «bérets verts " postés devant les mosquées de baščaršija et, après la prière, j'allais parfois avec eux boire un thé dans les cafés de ce vieux quartier commerçant. Un soir, je leur expliquais que le climat étrange qui enveloppait la ville m'épuisait et me glaçait le sang, et que j'avais décidé de rentrer à Belgrade. Ils insistèrent alors pour que je reste jusqu'au bajram ${ }^{10}$, m'expliquèrent que tout était normal, et qu'il n'y avait aucune raison d'avoir peur. En les écoutant, je regardais les kalachnikovs posées sur leurs genoux.

Au lendemain du référendum sur l'indépendance, nous avons été évacués vers Belgrade par un avion spécial de l'armée yougoslave, mis à disposition des diplomates venus observer le bon déroulement du vote. A quelques jours du bajram, j'ai pris un des derniers avions de la JAT à avoir quitté l'aéroport de Sarajevo. Le 5 avril 1992, j'ai vu sur mon écran de télévision la foule rassemblée devant le Parlement bosniaque, je l'ai vue brandir des portraits de Tito et applaudir naïvement les blindés de l'armée yougoslave, tenter de se protéger des tirs des snipers serbes postés sur les toits de l'Holiday Inn, puis partir les mains nues à l'assaut de l'hôtel. Ce jour-là, j'ai ressenti un tel mépris pour le 
peuple serbe que je n'ai pu m'empêcher de le cracher au visage de mes étudiants. Le lendemain, je me suis forcé à appeler les gens que je connaissais à Sarajevo, pour leur demander si je pouvais faire quelque chose. Ils m'ont remercié et dit de ne pas m'inquiéter, tout ceci ne durerait que quelques jours. J'ai raccroché en sachant que, de toute façon, je n'aurais rien pu faire.

Des années plus tard, le jour où les bombardements de l'OTAN ont commencé sur la Serbie, une amie belgradoise particulièrement chère m'envoya un e-mail maudissant le monde occidental, et moi avec. Je répondai que je comprenais sa fureur, que j'avais moi-même de sérieuses réserves sur ces bombardements, mais qu'elle ne devait pas oublier que la guerre n'avait pas commencé ce jour, mais huit ans auparavant. Après quelques vaines tentatives, nous avons cessé de communiquer.

17 Pour moi, tous ces souvenirs montrent une seule et même chose : la guerre est un événement si extraordinaire, une rupture si totale avec ce que nous connaissons, qu'elle est tout simplement incroyable. Nul ne peut, nul ne veut y croire avant qu'elle ne s'impose à lui. Dans ces circonstances, et quelles que soient par ailleurs les responsabilités ou les souffrances objectives des uns et des autres, chacun pourra se sentir agressé : le mauvais fusil est celui qui est braqué sur nous; le premier obus, et le pire de tous, est celui qui tombe sur notre maison. En d'autres termes, pour ceux qui la subissent, la guerre est une expérience fondamentalement subjective, située dans le temps et dans l'espace ${ }^{11}$.

Cette question des cadres spatiaux et temporels de référence, des perspectives choisies peut même conduire à des situations absurdes et déroutantes. Un jour que je discutais à Paris avec une amie serbe originaire de Sarajevo, celle-ci m'expliqua soudain que, làbas, les Serbes n'étaient pas les assiégeants, mais les assiégés. Bien qu'habitué aux propos fantasques et aux surenchères verbales des Serbes ${ }^{12}$, je lui faisais remarquer qu'elle délirait complètement. Elle me cita alors le cas de ses parents qui, vivant dans le quartier périphérique d'Ilida, devaient se méfier des tirs de mortier et de sniper venant à la fois de l'intérieur de la ville et du quartier de Butmir, situé au pied du mont Igman. Bien sûr, outre l'intensité variable des tirs, il existait une grande différence entre les habitants de Sarajevo et ceux d'Ilida : ces derniers pouvaient aller et venir en bus ou en voiture, et les camions leur apportant nourriture ou bois de chauffage n'avaient pas à franchir les lignes bosniaques. Mais un coup d'œil sur les lignes de front de l'époque ${ }^{13}$ permet aussi de comprendre que les Serbes d'Ilida aient pu se sentir encerclés, sans pour cela qu'il soit nécessaire de recourir à la légendaire " paranoïa serbe ».

Ma propre ubiquité mentale - je vivais en Serbie, et travaillais sur la BosnieHerzégovine - a eu des effets d'autant plus perturbants que s'y ajoutait un facteur aggravant: je m'intéressais à l'islam, et ce dans une perspective précise, à savoir l'analyse de l'islam politique telle qu'elle a été élaborée en France, au cours des années 1980, par Olivier Carré, Gilles Kepel et Olivier Roy ${ }^{14}$. Mon approche de l'islam bosniaque était donc très différente de celle qu'avaient pu en avoir auparavant les anthropologues William Lockwood, Cornelia Sorabji ou Tone Bringa ${ }^{15}$.

D'emblée, mon objet et mon angle de recherche m'ont placé dans une situation particulière. La première fois que je suis allé à Sarajevo, j'ai acheté dans un kiosque un numéro spécial de revue intitulé: "Est-ce que la Bosnie deviendra une république islamique? ${ }^{16}$. Il s'agissait en fait d'un recueil de textes et d'interviews, à la fin duquel figurait la désormais célèbre "Déclaration islamique » d'Alija Izetbegović, devenu peu auparavant Président de la Présidence collégiale bosniaque. Malgré mon serbo-croate 
encore hésitant, j'ai vite reconnu ce que l'on m'avait appris à reconnaître : une resucée des grands thèmes de la littérature islamiste. Ma première réaction fut de croire à un faux fabriqué par les Serbes. Ayant vérifié que ce n'était pas le cas, j'ai compris que je venais de tomber sur une mine, au double sens du terme: un filon extraordinaire à exploiter, et un objet très délicat à manier. Mais j'étais mal outillé pour le faire. Les deux cours que j'avais suivis à Sciences Po sur l'islam politique et sur les rapports entre religion et politique constituaient un bien maigre acquis. Le mémoire que j'avais rédigé à l'époque traitait de l'islam à Renault-Billancourt, un islam de travailleurs émigrés vieillissants, individualisé par son insertion dans un univers de travail répressif et un environnement social sécularisé ${ }^{17}$. Il n'est donc pas surprenant que mon objet m'ait explosé à la figure.

21 J'abordais cet objet par l'écrit et par le haut, d'une manière dont je n'allais jamais me déprendre vraiment et qui, au bout du compte, s'avèrerait dans le même temps très fructueuse et très glissante. Lors de mes séjours suivants à Sarajevo, j'ai focalisé mon attention sur Alija Izetbegović et son entourage, sur ce petit cercle d'islamistes projeté comme par miracle à la tête d'une communauté musulmane largement sécularisée, et sur la façon dont ce que je savais d'eux transparaissait dans leurs discours, ou influençait leurs choix ${ }^{18}$. J'accumulais les publications du Parti de l'action démocratique (SDA) et de la Communauté islamique, consultais à la bibliothèque Gazi Husrev-beg la presse religieuse des années 1930 et 1940, visitais l'équipe de "Muslimanski Glas ", qu'animaient de proches compagnons de route d'Izetbegovićn ${ }^{19}$, et négligeais tout le reste. A Belgrade, je rencontrais Darko Tanasković et Miroljub Jevtić, dont les commentaires sur les évolutions de l'islam yougoslave paraissaient dans la presse serbe. J'apprenais beaucoup du premier, et me rendais vite compte que le second était un fou dangereux ${ }^{20}$. Enfin, par l'intermédiaire d'un ami musulman vivant à Belgrade, je séjournais une semaine dans un village du Sandak, ce qui m'a permis de ne pas rester totalement ignorant des réalités de l'islam rural.

Dans le prolongement de ce travail, mon séjour à Sarajevo en mars 1992 avait pour but de suivre le déroulement du ramadan, et en particulier d'enregistrer une série de prêches à partir de laquelle j'espérais établir une typologie interne de l'islam bosniaque ${ }^{21}$.

Passantd'unemosquée à l'autre, j'ai entendu des prêches aux thèmes et aux accents bien différents, retrouvé au tabački mesdid (salle de prière des taneurs) certains rédacteurs de "Muslimanski Glas", que je découvrais dans leur rôle originel d'imam, côtoyé de jeunes fidèles assistant au cercle de discussion de la mosquée Ferhadija, prié prié ! - à la demande du gardien d'une autre mosquée, qui ne savait trop s'il devait laisser un non-musulman muni d'un magnétophone assister à la prière du soir. A tous, je dis merci, et je demande pardon.

Dans les mosquées aussi, l'ambiance était lourde et étrange. Jusqu'où pouvais-je aller, jusqu'où avais-je le droit d'aller dans ce monde qui m'acceptait, mais qui n'était pas le mien? Cette adoption, n'allait-elle pas se transformer un jour en lassitude ou en rancœur ? Et était-il bien normal que je glose avec quelques rédacteurs de « Muslimanski Glas " sur le caractère possible ou impossible, opportun ou inopportun, d'une transformation de la Bosnie en république islamique ? Je me suis souvent demandé, par la suite, pourquoi j'avais été ainsi accepté. Je crois que la bonhomie des Musulmans bosniaques, cette naïveté qu'ils se reprochent tant aujourd'hui, y est pour quelque chose. Je pense aussi que, à une époque où Sarajevo ne regorgeait pas d'étrangers, ils 
avaient plaisir à discuter avec un jeune Français parlant leur langue, et ayant une vague idée de leur religion. Et puis, il y avait la peur, cette peur qui nous réunissait : à un moment où nul ne savait de quoi le lendemain serait fait, et où les Musulmans attendaient tant de l'Europe, j'apparaissais sans doute, avec mon image d'employé de l'ambassade de France, comme un signe tangible que cet espoir n'était pas vain.

C'est dans ce contexte que, un soir, j'ai compris que je me trouvais là où je n'aurais pas dû être. Nous étions assis dans la mosquée Ferhadija, attendant l'imam qui, comme chaque soir, devait commenter un passage du Coran. En son absence, une discussion s'est engagée sur le plan Cutilheiro, un plan proposant le partage de la BosnieHerzégovine en cantons ethniques, et dont l'acceptation par Izetbegović conditionnait alors la reconnaissance internationale de la Bosnie-Herzégovine. Un jeune homme - le seul à l'époque de tout Sarajevo à arborer une tenue afghane - s'est levé, et a expliqué en substance que ce plan était bon, car il donnait aux Musulmans bosniaques un Etat plus grand que la Slovénie, mais que la question était dès lors la suivante: les Musulmans étaient-ils assez mûrs pour que, dans cet Etat, soit appliquée la charia ? Un autre homme, plus âgé, habillé à l'européenne, lui a alors rétorqué sur un ton ferme qu'il n'avait rien compris, que Alija avait raison de ne pas accepter le plan Cutilheiro, car il fallait d'abord obtenir la reconnaissance de la Bosnie-Herzégovine comme Etat unitaire, et ensuite seulement, car de toute manière les Musulmans n'étaient pas prêts, s'emparer du système éducatif pour préparer l'avènement d'une république islamique. Mes pensées ont commencé à se bousculer dans ma tête. Ce que je venais d'entendre, et que les Serbes m'affirmaient sur tous les tons depuis des mois, était-ce la réalité, où étais-je tombé dans un mauvais rêve serbe? Et comment réagiraient-ils, ces fiers orateurs, s'ils apprenaient qu'il y avait là, dans le public, un Français muni d'un passeport de service?

Le débat a sombré dans la confusion générale et, quand quelqu'un a dit qu'il ne fallait pas salir la réputation de l'islam comme l'avait fait l'imam Khomeiny, une partie de l'auditoire a quitté les lieux pour marquer son indignation. J'en ai profité pour filer. Le lendemain, je suis revenu et, face aux sourires gênés des personnes me connaissant, j'ai feint de n'avoir rien compris à la discussion de la veille. Mais cet incident a largement contribué à ma décision de quitter Sarajevo prématurément, et à celle de ne pas y revenir pendant la guerre ${ }^{22}$.

A mon retour en France en juin 1992, ma vision de la Bosnie-Herzégovine n'était donc pas tout à fait celle des militants qui découvraient les visages émaciés d'Omarska, et rejoignaient les comités Bosnie. J'avais du mal à ne voir dans Izetbegović qu'un doux grand-père un peu rêveur. Je ne supportais pas les parallèles tracés entre la Bosnie et ce que les intellectuels français savent généralement de la guerre d'Espagne, à savoir ce que les communistes ont bien voulu en dire. Je n'avais jamais croisé de ma vie Zlatko Dizdarević, Ibrahim Spahić ou le général Divjak ${ }^{23}$, et m'interrogeais sur leurs silences et leurs tics de vocabulaire : où était passé mon mauvais rêve serbe, et n'était-ce vraiment qu'un rêve? D'où sortait ce mot "agression ", et où avait disparu celui de "guerre civile", qui remplissait encore les pages du quotidien "Oslobodjenje $»^{24}$ quelques semaines auparavant? Un jour, croisant un universitaire parisien que j'avais connu à l'époque de Solidarnosc, celui-ci m'invita à rejoindre le mouvement de soutien à la Bosnie-Herzégovine, dont il était une des figures de proue. Je lui expliquais que, vu ce que je savais de spécifique sur les dirigeants bosniaques, le maximum que je pouvais 
faire était de me taire. C'est, en partie, ce que j'ai fait jusqu'à la parution du livre «Bosnie, anatomie d'un conflit » en $1996^{25}$.

Dans les trois mois qui séparent mon séjour à Sarajevo de mon retour à Paris, la guerre n'a pas rendu mon travail plus difficile, elle en a fait une tâche absurde et coupable. Pendant toute la durée de la guerre, je n'ai pas su quoi faire de ce que je savais, et du reste, que savais-je au juste? Les rares articles que j'ai publiés m'ont valu les appels intéressés des barbouzes de France et de Navarre, et des équipes de la télévision serbe. Je suis allé voir Rémy Leveau, mon premier directeur de thèse, en lui demandant de changer de sujet, je l'ai accusé de m'avoir fourré dans cette impasse. Je m'imprégnais de " Muslimanski Glas », devenu entre temps « Ljiljan » ("Le lys »), reconnaissais le style des uns et des autres, traquais leurs sous-entendus, maudissais leurs détours. Je me réveillais la nuit en me demandant si j'avais bien lu ce que j'avais lu, entendu ce que j'avais entendu, compris ce que j'avais compris. Je pensais à cette femme qui, lorsque je m'étais présenté au tabački mesdid, avait sèchement dit que les orientalistes étaient tous des ennemis de l'islam. Plus tard, amadouée sans doute par mon assiduité, elle m'avait offert un tespih (chapelet). Je me demandais ce qu'elle était devenue, et ce qu'elle penserait de moi. Mon enfer à moi tenait tout entier dans ma tête.

29 A la pression des discours médiatiques et militants, au poids des souvenirs et des remords s'ajoutait un rapport complexe à mon milieu professionnel le plus proche. Mes deux directeurs de thèse successifs, Rémy Leveau et Gilles Kepel, n'ont jamais cessé de me faire confiance, et je dois à leur soutien amical mais exigeant le fait d'avoir achevé ma thèse. Mais, plus généralement, je me trouvais en porte-à-faux avec ceux travaillant sur l'islam politique, et devais prendre à contre-pied un certain nombre de discours et d'usages.

Le grand mérite de ceux qui ont étudié l'islam politique à partir des années 1970 est d'avoir rompu avec ce que l'orientalisme classique peut avoir de dominateur et de réducteur, pour montrer que les sociétés musulmanes étaient des sociétés en mouvement, traversées par une multiplicité de discours et d'acteurs. Face à la diabolisation des mouvements islamistes par les médias occidentaux, ils ont montré que ces mouvements étaient aussi des vecteurs de modernité, ont insisté sur leurs motivations sociales et leurs potentialités démocratiques, se sont efforcés de les "banaliser» en soulignant leurs liens avec les idéologies nationalistes, ou leurs fonctions tribunitiennes dévolues en d'autres lieux au parti communiste. Cette attitude a $\mathrm{pu}$ engendrer parfois une certaine complaisance, ou une routine intellectuelle consistant à attendre que le discours médiatique se réamorce pour se livrer une fois de plus à sa déconstruction. Mais là n'est pas l'important. J'adhère pour l'essentiel aux analyses de l'islamisme élaborées à partir des années 1970, et ma thèse de doctorat n'est rien de plus que leur application au cas bosniaque.

Le problème est que j'avais affaire à un objet atypique - une minorité islamiste parvenue au pouvoir en l'absence de toute mobilisation islamiste de masse -, et à un traitement médiatique de cet objet tout aussi atypique: le plus souvent, en effet, l'islamisme bosniaque n'était pas diabolisé, mais nié ou passé sous silence. Dans son ouvrage de référence sur la Bosnie, Noel Malcolm considère ainsi que la "Déclaration islamique " constitue "de simples articles de foi auxquels adhère tout musulman sincère $»^{26}$ et, dans leur majorité, les médias occidentaux n'ont cessé de dénier toute «menace islamiste » en Bosnie en insistant sur le caractère européen et tolérant de l'islam bosniaque. Pour justifier mon propre travail, je devais contrer cet orientalisme 
d'un genre nouveau, qui identifiait les Musulmans bosniaques à Izetbegović, et opposait en bloc l'islam bosniaque à un autre islam implicite, non-tolérant car non-européen. Il me fallait donc prouver l'existence d'un courant islamiste en Bosnie-Herzégovine, souligner qu'il ne se réduisait ni à un proto-nationalisme, ni à un néo-communisme, aller au-delà de cette modernité qu'il partageait avec l'ensemble de la société bosniaque, pour montrer en quoi il ne se confondait pas avec elle. Trouvant de la modernité au cœur des mouvements islamistes, mes maitres s'en sont servis pour les rendre plus intelligibles. Trouvant de l'islamisme au cœur de l'Europe moderne, j'aurais voulu m'en servir pour la rendre plus problématique. Mais je n'y suis pas parvenu.

\section{NOTES}

1.. Bien sûr, ce constat vaut également pour les personnes engagées en faveur de la Bosnie-Herzégovine, avec qui je me suis souvent trouvé en profond désaccord, et dont certains m'ont vivement attaqué dans leurs écrits. Sur les interrogations et les dissensions morales des milieux français ayant soutenu la cause bosniaque, voir entre autres B-H. Levy, «La liste, et après », La règle du jeu, $\mathrm{n}^{\circ} 14$, septembre 1994, pp. 90-124; F. Martel, « Pour servir à l'histoire de notre défaite. L'élite' intellectuelle et morale' française et la guerre en ex-Yougoslavie (1991-1994) », Le messager européen, n8, 1994, pp. 127-154 ; E. Wallon, « La guerre de Sarajevo a vraiment eu lieu. Notes sur l'engagement des artistes et des intellectuels », Les temps modernes, ${ }^{\circ} 587$, mars-mai, 1996, pp. 374-399 ; J-F. Narodetzki, Nuits serbes et brouillards occidentaux. Introduction à la complicité de génocide, Paris, L'Esprit frappeur, 1999.

2. . Sur l'épreuve que peut constituer une recherche de terrain dans un pays en guerre, voir C. Nordstrom / A. Robben (ed.), Fieldwork Under Fire. Contemporary Studies of Violence and Survival, Berkeley, University of California Press, 1995. Sur le cas spécifique de l'ex-Yougoslavie, voir entre autres M. Povrzanović, « Etnologija rata : pisanje bez suza? » [L'ethnologie de la guerre : écrire sans larme ?], Etnolo(ka tribina, $\mathrm{n}^{\circ} 15$ (1992), pp. 61-80, ainsi que la thèse non-publiée de Ivana Maček sur Sarajevo assiégée (I. Maček, War Within. Everyday Life in Sarajevo Under Siege, University of Uppsala, 2000).

3. . G. Orwell, Hommage à la Catalogne, Paris, Champ libre, 1984.

4. . T. Todorov, Face à l'extrême, Paris, Seuil, 1994.

5. . «Borba » (« Combat») était le principal quotidien belgradois d'opposition, proche du Premier ministre fédéral Ante Marković, et sans doute une des sources d'information les plus complètes et les plus fiables de l'espace yougoslave. En décembre 1994, le pouvoir serbe parvint à s'emparer de « Borba ». La plupart des journalistes quittèrent alors ce journal devenu un simple torchon, et créèrent le quotidien indépendant «Naŝa borba » (« Notre combat »), qui disparut quelques années plus tard. 6. . J'ai à l'époque décrit cette situation dans un article publié sous pseudonyme. Voir B. Useljeniĉki, «Les 'traîtres'. Déserteurs et pacifistes dans la Serbie de Miloŝević », Les temps modernes, ${ }^{\circ}$ 545-546, décembre 1991-janvier 1992, pp. 311-320. 
7. . Pour une analyse fine de l'atmosphère qui régnait à Belgrade pendant les années de guerre, et du rétrécissement des horizons intellectuels qui en a résulté, voir E. Gordy, The Culture of Power in Serbia. Nationalism and the Destruction of Alternatives, University Park, The Pennsylvania State University Press, 1999.

8. . Sur le mouvement anti-guerre à Sarajevo avant avril 1992, voir la thèse de doctorat non-publiée de Neven Andjelić sur la Bosnie-Herzégovine des années 1980 (N. Andjelić, Bosnia-Herzegovina : Politics and Society at the End of Yugoslavia, University of Sussex, 1999). Un bref résumé de cette thèse a été publié dans la revue «Balkanologie ». Voir N. Andjelić, «L'évolution de la société civile dans la Bosnie-Herzégovine d'avant-guerre », Balkanologie, vol. IV, $n^{\circ} 1$, septembre 2000, pp. 27-51.

9. J'étais venu à Sarajevo avec Ariane, ma compagne, pour lui montrer cette jolie ville, et en lui expliquant doctement qu'il n'y avait aucun risque, car les seuls incidents envisageables se dérouleraient à la campagne.

10. . Les festivités du ramazanski bajram (en arabe : 'id al-fitr) marquent la fin du mois de ramadan et durent trois jours.

11. Sur la guerre comme expérience située dans l'espace, voir entre autres M. Povrzanović, « Identities in War : Embodiments of Violence and Places of Belonging », Ethnologia Europea, n²7, 1997, pp. 153-162. Cette inscription dans l'espace et dans le temps de toute expérience de la guerre explique les effets de filiation ethnique et d'appartenance générationnelle auxquels les chercheurs travaillant sur l'espace yougoslave n'ont pas échappé. Ainsi, j'ai toujours été surpris de voir à quel point les personnes ayant commencé à étudier la Yougoslavie avant et après 1991 avaient une perception différente des événements, et je suis sorti de ce constat prématurément vieilli. A ce sujet, voir J. Allcock, «Involvement and Detachment : Yugoslavia as an Object of Scholarship ", Journal of Area Studies, $\mathrm{n}^{\circ} 3$, 1993, pp. 144-160.

12. Sur l'imaginaire politique des Serbes au cours des années 1990, voir en particulier les différents ouvrages de I. Ĉolović, Bordel ratnika [Le bordel des guerriers], Belgrade : XX. vek (1993) ; Pucanje od zdravlja [Exploser de bonne santé / Exploser hors des limites de la santé], Belgrade, Krug, 1994 ; Politika simbola : ogledi o političkoj antropologiji [Politique du symbole : essais d'anthropologie politique], Nelgrade : Radio B 92 (1997), ainsi que la thèse non-publiée de Marko ivković (M. ivković, Serbian Stories of Identity and Destiny in the 1980s and 1990s, University of Chicago, 2001).

13. . Voir par exemple la carte du siège de Sarajevo reproduite en page 93 de mon ouvrage « Bosnie, anatomie d'un conflit » (X. Bougarel, Bosnie. Anatomie d'un conflit, Paris, La Découverte, 1996).

14. . Voir entre autres O. Carre / G. Michaud, Les Frères musulmans, Paris, Archives Gallimard, 1983 ; G. Kepel, Le Prophète et Pharaon, Paris, La Découverte, 1984 ; O. Roy, L'Afghanisatan, islam et modernité politique, Paris, Seuil, 1985 ; O. Carre / P. Dumont (dir.), Radicalismes islamiques, Paris, L'Harmattan, 1986 ; G. Kepel / Y. Richard (dir.), Intellectuels et militants de l'islam contemporain, Paris, Seuil, 1990 ; O. Roy, L'échec de l'islam politique, Paris, Seuil, 1992.

15. . Voir W. Lockwood, European Moslems. Economy and Ethnicity in Western Bosnia, New-York / London, Academic Press, 1974 ; C. Sorabji, Muslim Identity and Islamic Faith in Socialist Sarajevo, University of Cambridge, 1988, thèse de doctorat nonpubliée ; T. Bringa, Being Muslim the Bosnian Way. Identity and Community in a Central Bosnian Village, Princeton, Princeton University Press, 1995. 
16. . «Da li će Bosna biti islamska republika? ». Je ne retrouve plus ce numéro de revue dans mes archives et je suppose donc que j'ai dû m'en débarrasser entre temps.

17. . Voir X. Bougarel / P. Diallo, « Les travailleurs musulmans à Renault-Billancourt : le repli », Revue européenne des migrations internationales, vol. VII, n³, 1991, pp. 77-89. 18. Sur l'histoire de cette mouvance islamiste jusqu'à la création du Parti de l'action démocratique (SDA) et son accession au pouvoir en 1990, voir X. Bougarel, « Un courant panislamiste en Bosnie-Herzégovine », dans G. Kepel (dir.), Exils et royaumes. Les appartenances au monde arabo-musulman aujourd'hui, Paris, Presses de la FNSP, 1994, pp. 275-299.

19. . « Muslimanski Glas » (« La voix musulmane »), organe officieux du SDA, était dirigé par Demaludin Latić, une des personnes condamnées avec Alija Izetbegović pour «fondamentalisme islamique » en 1983, et une large partie de son équipe rédactionnelle était constituée d'anciens élèves de la medressa de Sarajevo.

20. . Darko Tanasković était professeur d'arabe, de turc et de philologie orientale à la Faculté de lettres, et Miroljub Jevtić enseignant à la Faculté de sciences politiques de l'université de Belgrade. Ils sont tous deux accusés par Norman Cigar, un expert américain d'origine croate, de figurer parmi les artisans intellectuels du génocide contre les Musulmans de Bosnie-Herzégovine (voir N. Cigar, Genocide in Bosnia. The Policy of Ethnic Cleansing, College Station, Texas A\&M University Press, 1995). Dans un article paru en 1998, j'ai pour ma part tenté de montrer en quoi les positions intellectuelles et les choix politiques de Darko Tanasković, que je suis loin d'approuver sans réserve, doivent être distingués des délires monomaniaques de Miroljub Jevtić. Voir X. Bougarel, «L'islam et la guerre en Bosnie-Herzégovine : l'impossible débat? ", L'Autre Europe, n³6-37, hiver 1998 / 1999, pp. 106-116.

21. . Voir X. Bougarel, « Discours d'un ramadan de guerre civile », L'Autre Europe, n²6-27, hiver 1992 / 1993, pp. 171-197.

22. . Pendant toute la guerre, je me suis demandé comment réagiraient ceux que j'avais connu à Sarajevo avant la guerre quand ils me reverraient. En septembre 1993, de passage à Ljubljana, je visitais la rédaction de « Muslimanski Glas » qui s'y était établie en partie. L'accueil cordial que je reçus me montra que j'avais été exagérément méfiant. Toutefois, c'est aussi à Ljubljana que j'ai connu la situation la plus menaçante pour ma propre sécurité. Visitant le siège local du SDA, au moment même où ce parti était ébranlé par une crise interne très violente, je m'y suis trouvé retenu de force par les permanents locaux du parti, qui s'étaient mis en tête de me faire dire pour quels services secrets je travaillais. Relâché au bout d'une bonne heure, je décidais de ne pas rester bloqué sur cette expérience désagréable et, après avoir repris mes esprits à la terrasse d'un café, me rendais à la rédaction du quotidien « Oslobodjenje » (voir note 24). Je tombais en pleine célébration du cinquantième anniversaire de ce journal, et c'est donc ragaillardi et passablement éméché que, le soir même, je prenais un bus pour Zagreb. Par la suite, je me suis souvent demandé comment se serait terminée ma mésaventure si elle n'avait pas eu lieu dans la capitale slovène, mais dans une ville bosniaque telle que Zenica, Tešanj ou Zavidovići.

23. . Zlatko Dizdarević, Ibrahim Spahić et Jovan Divjak sont trois figures francophones de Sarajevo assiégée. Zlatko Dizdarević était journaliste au quotidien « Oslobodjenje ", et est notamment l'auteur d'un «Journal de guerre » publié en français (Z. Dizdarević, Journal de guerre, Paris, Spengler, 1993). Ibrahim Spahić était le directeur du festival d'hiver de Sarajevo, auquel ont participé plusieurs troupes de théâtre françaises pendant les années de siège. Le général Jovan Divjak était membre de l'état-major de 
l'armée bosniaque et, en tant qu'officier supérieur serbe, était censé attester le caractère multiethnique de cette armée.

24. . « Oslobodjenje » (« Libération »), principal quotidien bosniaque proche du Parti social-démocrate (ex-communiste), a été un des symboles de la résistance de Sarajevo. Les bâtiments abritant sa rédaction et son imprimerie, situés près de la ligne de front, ont été presque entièrement détruits par l'artillerie serbe.

25. . X. Bougarel, Bosnie, anatomie d'un conflit, op. cit.

26. . N. Malcolm, Bosnia, a Short History, London, Papermac, 1994, p. 220.

INDEX

Index géographique : Balkans, Bosnie-Herzégovine

Mots-clés : religion, guerre 